\title{
On the Performance of Blind Chip Rate Estimation in Multi-Rate CDMA Transmissions Using Multi-Rate Sampling in Slow Flat Fading Channels
}

\author{
Siavash GHAVAMI, Bahman ABOLHASSANI \\ School of Electrical Engineering, Iran University of Science and Technology, Tehran, Iran \\ Email: sghavami@ee.iust.ac.ir,abolhassani@iust.ac.ir
}

Received March 7, 2009; revised March 19, 2009; accepted March 20, 2009

\begin{abstract}
This paper considers blind chip rate estimation of DS-SS signals in multi-rate and multi-user DS-CDMA systems over channels having slow flat Rayleigh fading plus additive white Gaussian noise. Channel impulse response is estimated by a subspace method, and then the chip rate of each signal is estimated using zero crossing of estimated differential channel impulse response. For chip rate estimation of each user, an algorithm which uses weighted zero-crossing ratio is proposed. Maximum value of the weighted zero crossing ratio takes place in the Nyquist rate sampling frequency, which equals to the twice of the chip rate. Furthermore, bit time of each user is estimated using fluctuations of autocorrelation estimators. Since code length of each user can be obtained using bit time and chip time ratio. Fading channels reduce reliability factor of the proposed algorithm. To overcome this problem, a receiver with multiple antennas is proposed, and the reliability factor of the proposed algorithm is analyzed over both spatially correlated and independent fading channels.
\end{abstract}

Keywords: Multi-Rate Sampling, Chip Time, Multi-Rate CDMA, Blind Estimation

\section{Introduction}

Direct-sequence code division multiple accesses (DSCDMA) systems are nowadays of increasing importance in wireless cellular communications because of their inclusion in most of the proposals about both terrestrial and satellite based standards for third-generation (3G) wireless networks [1,2]. On the other hand, direct sequence spread spectrum (DS-SS) signals are well-known and are used in secure communication for their low probability of interception, their statistics are similar to those of noise; furthermore, they are usually transmitted below the noise level.

One of the salient features of 3G cellular systems is the capability of supporting transmission data as diverse as voice, packet data, low-resolution video, and compressed audio. Since these heterogeneous services pro- duce digital information streams with different data rates, their implementation requires the use of multi-rate CDMA systems where each user may transmit his data at one among a set of available data rates. An easy way to view the multi-rate CDMA transmission is to consider the variable spreading length (VSL) technique where all users employ sequences with the same chip period; moreover, the data rate is tied to the length of the spreading code of each user. Another way to view a multi-rate CDMA transmission is to consider a constant spreading length where users employ sequences with different chip periods. In general, chip time and spreading sequence length of users can be selected variable.

Two recent systems are often applied in military systems based on spread spectrum. In the literature, different methods have been presented for chip time estimation, many number of those methods are based on cyclic cumulant method, which have been presented in litera- 
tures [3-7]. Cyclic cumulant method in multi-rate and multi-user system doesn't exhibit good performance because cyclic frequencies of different users overlap and chip time estimation of different users is difficult. In [8] we proposed a blind chip time estimation algorithm based on multi-rate signal processing.

In this paper, blind chip time estimation technique is considered, which is based on channel impulse response estimation using singular value decomposition of estimated received signal covariance matrix. For spreading sequence estimation in slow flat Rayleigh fading channel plus additive white Gaussian noise (AWGN), length of code must be determined. Bit time and chip time must be estimated, for length of code estimation. In the proposed method in this paper, chip time is determined using multi-rate sampling of channel impulse response. Maximum number of zero crossing in differential of channel impulse response take places in sampling frequency twice of the chip rate. Therefore, number of zero crossing is obtained as a function of sampling rate. Then bit time is determined using fluctuations of correlation estimator. Code length of each user is determined using their corresponding chip times and bit times. Finally, performance of the proposed method is analyzed in fading channels and a receiver with multiple antennas is proposed for performance improvement in fading channels. Estimated parameter using this method is useful and applicable in noted systems in pervious paragraph in very low signal to noise ratio (SNR) (negative SNR in dB). This needs no prior knowledge about transmitter in the receiver side; it is typically the case in blind signals interception in the military field or in spectrum surveillance.

The remaining of this paper is organized as follows. In Section 2, System model are introduced. In Section 3, subspace methods for channel impulse response estimation are reviewed. Section 4 proposed blind chip time estimation based on multi-rate sampling. Blind bit time estimation is considered in Section 5. In Section 6, the performance of the proposed chip rate estimation algorithm will be analyzed over spatially correlated and non correlated flat fading channels. Simulation results are expressed in Section 7. Finally conclusions are performed in Section 8.

\section{System Model}

We consider the down link scenario of a multi-rate DS-CDMA network. The base station transmits signals and a mobile station receives a combined signal of $K$ active users, which is given by

$$
r(t)=\sum_{i=1}^{S} \sum_{k=1}^{K_{i}} \sum_{j=-\infty}^{+\infty} A_{k} d_{k}[j] h_{k, i}\left(t-j T_{s_{i}}-\tau_{k, i}\right)+n(t),
$$

where $A_{k}, d_{k}[j], h_{k}(t), T_{s_{i}}$ and $\tau_{k, i}$ are the received amplitude, $j^{\text {th }}$ data symbol, convolution of channel impulse response and spreading sequence waveform, symbol time and delay of $k^{\text {th }}$ user in $i^{\text {th }}$ rate respectively. Also, $K_{i}$ is number of active users in $i^{\text {th }}$ rate, $S$ is the number of available rates, and $n(t)$ is additive white Gaussian noise. In the down link scenario, $\tau_{k, i} \mathrm{~s}$ are equal, and the $h_{k, i}(t)$ is expressed by

$$
h_{k, i}(t)=L_{i}^{-1 / 2} \sum_{j=0}^{L_{i}-1} c_{k, i} p_{i}\left(t-j T_{c_{i}}\right)
$$

where $L_{i}$ is the spreading sequence length, $T_{\mathrm{c}_{i}}=$ $T_{s_{i}} / L_{i}$ is the chip time of $i^{\text {th }}$ rate, $p_{i}(t)$ is explained in the next paragraph, $c_{k, i}[m]$ is the value of the $m^{\text {th }}$ chip and $i^{\text {th }}$ rate with $\left|c_{k, i}[m]\right|=1$. Data symbols $\left\{d_{k}[j]\right\}$ of different users have independent identically distributions (i.i.d.). In this paper, channel model is considered slow flat Rayleigh fading plus AWGN. We assume that the channel in the duration of one processing window remains approximately constant.

In (2), $p_{i}(t)$ is the total channel impulse response of the $k^{\text {th }}$ user in $i^{\text {th }}$ rate, it is convolution of the transmitter filter $e(t)$, channel filter $s(t)$ and receiver filter $g(t)$ i.e.

$$
p_{i}(t)=e(t) * s(t) * g(t)
$$

where these filters have unit energy. $s(t)=\beta_{k}(t) e^{j \varphi_{k}(t)}$ $\delta(t), \quad \beta_{k}$ s have Rayleigh distribution and $\varphi_{k}$ s have uniform distribution, $*$ operation denotes convolution operation. We assume that

1) Channel model is slow flat Rayleigh fading plus AWGN.

2) Signal power is lower than noise power (SNR $<0$ in $\mathrm{dB})$.

3) A symbol time is equal to the spreading sequence length, i.e. $T_{c_{i}}=T_{s_{i}} / L_{i} \quad$ (i.e. short spreading code).

4) Symbols have zero mean and are uncorrelated.

\section{Estimation of Channel Impulse Response}

Covariance matrix of the received signal can be written as

$$
\mathbf{R}_{r}=E\left\{\mathbf{r r}^{H}\right\}=E\left\{\mathbf{x x}^{H}\right\}+E\left\{\mathbf{n n}^{H}\right\}=\mathbf{R}_{x}+\mathbf{R}_{n}
$$

where $\mathbf{r}$ is vector of received signal, $\mathbf{x}$ is vector of received signal when noise is absent,$H$ denotes hermitian operation, and $\mathbf{n}$ is vector of additive white Gaussian 
noise. Furthermore, $\mathbf{R}_{x}$ and $\mathbf{R}_{n}$ show covariance matrix of the received signal when noise is absent and noise covariance matrix, respectively. Covariance matrix of the received signal can be written as [9]

$$
R=\sigma_{n}^{2}\left\{\sum_{k=0}^{K-1} \beta_{k}\left\{\left(1-\alpha_{k}\right) \mathbf{v}_{k}^{0}\left(\mathbf{v}_{k}^{0}\right)^{*}+\alpha_{k} \mathbf{v}_{k}^{-1}\left(\mathbf{v}_{k}^{-1}\right)^{*}\right\}+\mathbf{I}\right\}
$$

where, $\sigma_{n}^{2}$ is noise variance, $\alpha_{k}=\tau_{k} / T, \beta_{k}=\sigma_{s i_{(k, i)}}^{2} T$ $/\left(\sigma_{n}^{2} T_{s_{i}}\right)$, in which $\sigma_{s i g_{(k, i)}}^{2}$ is power of signal of $k^{\text {th }}$ user in $i^{\text {th }}$ rate. $\mathbf{v}_{k}^{0}$ and $\mathbf{v}_{k}^{-1}$ are normalized eigenvector of the estimated covariance matrix of received signal, they correspond to $k^{\text {th }}$ asynchronous user in $i^{\text {th }}$ rate, $\boldsymbol{T}$ is the sampling period, and $\mathbf{I}$ is the identity matrix.

In the downlink scenario, when signal of one user are synchronized all user are synchronized $\left(\alpha_{k} \mathrm{~s}=0\right)$ and covariance matrix of the received signal is given by

$$
R=\sigma_{n}^{2}\left\{\sum_{k=1}^{K-1} \beta_{k}\left(\mathbf{v}_{k} \mathbf{v}_{k}^{*}\right)+\mathbf{I}\right\}
$$

\section{Blind Chip Rate Estimation}

In singular value decomposition of the received signal covariance matrix, if the chip rate is less than half of the sampling frequency, estimated eigenvector of the received signal covariance matrix (corresponds to maximum eigenvalue) is the convolution of $e(t), s(t)$, $g(t)$ and spreading sequence of synchronized user. If the impulse response of transmitter and receiver filters have been assumed ideal, and channel is constant in the duration of one processing window, estimated eigenvector will be the multiplication of spreading sequence of synchronized user in constant value. Hence for estimating spreading sequence from estimated impulse response of channel by subspace method i.e. $\mathbf{v}_{k}$, chip rate must be known exactly.

Sampling ratio is defined as

$$
m=\frac{F_{s}}{R_{c}}
$$

The total impulse response of the channel related to each user, (2), can be written as follows

$$
h_{k, i}(t)=\sum_{j=0}^{m L_{i}-m} c_{k, i} p\left(t-j \frac{T_{\mathrm{c}}}{m}\right) .
$$

If the sampling frequency is known, by estimation $h_{k, i}(t)$ using eigenvector of synchronized signal and relation between sampling time and chip time (i.e.
$T_{e}=T_{c} / m$ ), we can obtain chip rate of the signal. Hence we focus on the estimation of sampling ratio $(S R)$.

First, we calculate sign of $h_{k, i}(t)$ to obtain the normalized spreading sequence, it is performed due to binary phase shift keying (BPSK) spreading assumption of transmit signal. Sign of $h_{k, i}(t)$ is given by

$$
\operatorname{sgn}\left(h_{k, i}(t)\right)=\sum_{j=0}^{m L_{i}-m} c_{k, i}\left[u\left(t-(j+1) \frac{T_{\mathrm{c}}}{m}\right)-u\left(t-j \frac{T_{\mathrm{c}}}{m}\right)\right]
$$

where $u(t)$ is the unit step function. Then, the differential of $\operatorname{sgn}\left(h_{k, i}(t)\right)$ is calculated with respect to $t$ to estimate the number of zero crossings of the spreading sequence, which is given by

$$
\frac{d \operatorname{sgn}\left(h_{k, i}(t)\right)}{d t}=N^{-1 / 2} \sum_{j=0}^{m L_{i}-m} c_{k, i}[m] \delta\left(t-j \frac{T_{\mathrm{c}}}{m}\right)
$$

$d \operatorname{sgn}\left(h_{k, i}(t)\right) / d t$ includes impulse sequences by zero crossings at times $t=j T_{\mathrm{c}} / \mathrm{m}$. It is obvious that in sampling frequencies greater than the Nyquist rate, by increasing the sampling frequency, number of zero crossings on differential of spreading sequence remains constant. Figure 1(a) shows a sequence with length of 63, and Figure 1(b) shows the same sequence with the $S R$ of 4. Figure 1(c) shows the differential of the sequence shown in Figure 1(a) and Figure 1(d) is similar to Figure 1(c) for differential of sequence which has been shown in Figure 1(b). It can be seen in this figure, the number of zero crossings remains constant in differential of each sequence by over sampling greater than the Nyquist rate. Figure 2(a) shows the number of zero crossings of the differential of sequence in terms of different sampling frequencies. Bandwidth of signal is considered $10 \mathrm{MHz}$ and it can be seen the number of zero crossings remains constant for sampling frequency greater than $20 \mathrm{MHz}$. Zero crossing ratio is defined as the following

$$
Z C R=\frac{\text { Number of zero crossings }}{\text { Length of diffrentiated Sequence }}
$$

Figure 2(b) shows the number of zero crossings ratio of the differential sequence in terms of sampling frequency. It is obvious that for sampling frequencies greater than the Nyquist rate, ZCR reduces by increasing the sampling frequency and the maximum value of $Z C R$ occurs at the sampling frequency equal to the chip rate of the sequence (i.e. length of 63). But for sampling frequencies less than the Nyquist rate, for loss of some data samples and decreasing length of differential 


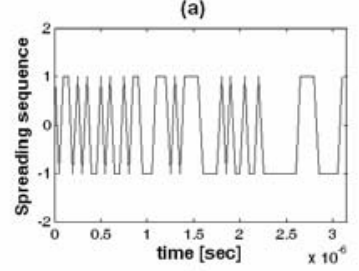

(c)

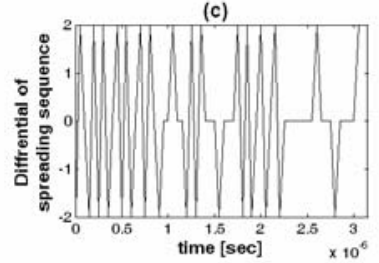

(b)

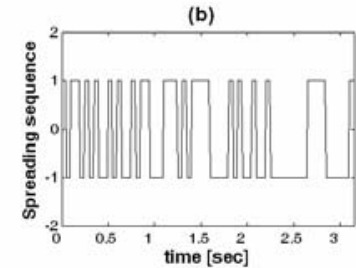

(d)

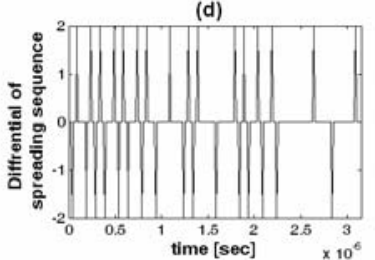

Figure 1. (a) Spreading sequence (length of 63). (b) Over sampled spreading sequence (length of 63 ) and $S R=4$. (c) Differential of Spreading sequence (length of 63). (d) Differential of Over sampled spreading sequence (length of 63) and $S R=4$.

sequence, ZCR may be greater than ZCR corresponding to the Nyquist rate. In Figure 2(b) an example of this situation can be seen. Sampling frequencies less than Nyquist rate losses some of data samples and decrease length of differential of sequence, it causes ZCR increases for sampling frequency lower than the Nyquist rate. Since, by weighting $Z C R$, it is possible to reduce $Z C R$ for sampling frequencies less than the Nyquist rate. Therefore, we propose weighted zero crossing ratio, which is defined as

$$
W Z C R=\text { The Number of zero crossings } \times Z C R
$$

Figure 2(c) shows weighted zero crossing ratio in terms of frequency sampling, it can be seen the maximum of WZCR corresponds to the sampling frequency is equal to the chip rate.

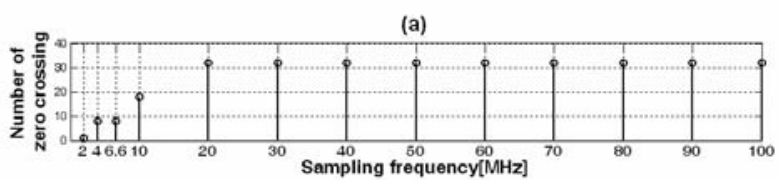

(b)

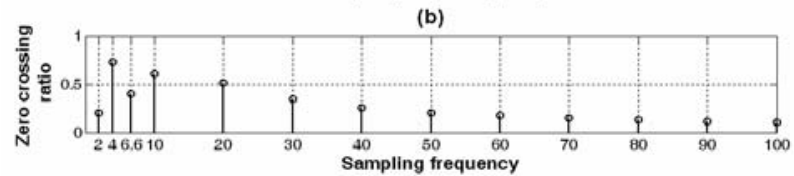

(c)

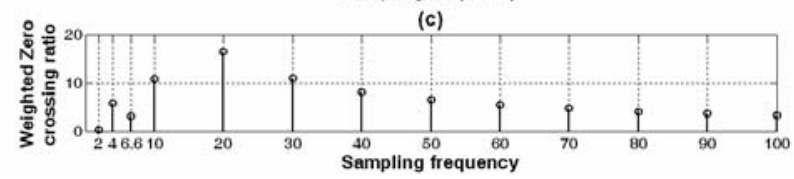

Figure 2. (a) Number of Zero crossing in terms of sampling frequency in MHz. (b) Zero crossing ratio in terms of sampling frequency. (c) Weighted zero crossing ratio in terms of sampling frequency.

Increasing the code length increases the computational complexity of subspace decomposition of the received signal covariance matrix due to increasing the dimension of covariance matrix, instead, output SNR in output of covariance matrix estimator increases by the factor of $N_{2} / N_{1}$, as,

$$
\frac{S N R_{\text {out }_{2}}}{S N R_{\text {out }_{1}}}=\frac{N_{2}}{N_{1}}
$$

where $N_{2}$ and $N_{1}$ are spreading sequence lengths corresponds to $S N R_{\text {out }_{2}}$ and $S N R_{\text {out }_{1}}$, respectively. Therefore by increasing code length it is possible to estimate spreading sequence of active users in lower SNR values.

\section{Blind Bit Rate Estimation}

For blind bit rate estimation, we use fluctuation of correlation estimator [10]. To compute the fluctuations, we divide the received signal into $M$ temporal windows with duration of $T_{F}$ for each window. Then, a correlation estimator is applied to each window prior to the computation. For the $m^{\text {th }}$ window, an estimation of the autocorrelation for any signal $r(t)$ is given by

$$
\hat{R}_{r}^{m}(\tau)=\frac{1}{T_{F}} \int_{0}^{T_{F}} r_{m}(t) r_{m}^{*}(t-\tau) d t
$$

where $r_{m}(t)$ is the signal sample over the $m^{\text {th }}$ window. Replacing $r_{m}(t)$ in (14) with the $m^{\text {th }}$ window of $r(t)$, and taking expectation of $\hat{R}_{r}^{m}(\tau)$ for $M$ windows, lead to the following equation, which can be used for both the uplink (asynchronous user) and downlink (synchronous) transmission,

$$
\hat{R}_{r}(\tau)=\hat{R}_{s}(\tau)+\hat{R}_{n}(\tau)
$$

where $\hat{R}_{n}(\tau)$ is autocorrelation of noise and

$$
\widehat{R}_{s}(\tau)=\sum_{i=0}^{S-1} \sum_{k=0}^{K_{i}} \hat{R}_{s_{k, i}}(\tau) .
$$

In (16), $\hat{R}_{s_{k, i}}(\tau)$ is the noise-unaffected estimation of the autocorrelation for the $(k, i)^{\text {th }}$ signal. Indeed, since the fluctuations are computed from many randomly-selected windows, they do not depend on the relative delays of signals. The use of $M$ windows allows us to estimate the second-order moment of the estimated correlation $\hat{R}_{r}^{m}(\tau)$ as

$$
\Phi(\tau)=\hat{E}\left\{\left|\hat{R}_{r}(\tau)\right|^{2}\right\}=\frac{1}{M} \sum_{m=0}^{M-1}\left|\hat{R}_{r}(\tau)\right|^{2}
$$


where $\hat{E}\{\cdot\}$ is the estimated expectation of $(\cdot)$. Hence, $\Phi(\tau)$ is a measure of the fluctuations of $\hat{R}_{r}^{m}(\tau)$. The difference between the successive and equal amplitude peaks in (16) determines symbol time for each rate [10].

\section{Performance of the Proposed Chip Rate Estimation Algorithm}

In the proposed chip rate estimation algorithm, if the eavesdropping receiver fallen in the deep fading; the estimated chip rate is not valid. In this section, effect of fading channel is analyzed on the performance of the proposed chip rate estimation algorithm. This analysis is first done for a receiver using a single antenna. Second, the analysis is done for a receiver using multiple antennas to overcome fading.

The threshold value of SNR for chip rate estimation algorithm is defined as $S N R_{\text {min }}$, which is the minimum SNR for achieving a reliability factor $(R F)$ of chip rate estimation greater than $0.99 . S N R_{\min }$ is obtained using computer simulation in non-fading channels and its value depends directly on the spreading factor.

By assuming Rayleigh distribution for the channel, the probability that the received signal voltage $r$ is less than a given the threshold $R$, which is corresponding value of $S N R_{\min }$, found from the following equation

$$
\operatorname{Pr}(r \leq R)=\int_{0}^{R} p(r) d r=1-\exp \left(-\rho^{2}\right)
$$

where $p(r)$ is the probability density function (PDF) of the Rayleigh distribution, and $\rho=R / R_{r m s}$ is the value of the specified level $R$, normalized to the local rms amplitude of the fading duration envelope, hence $\rho^{2}=S N R_{\min } / S N R$. The chip rate estimation algorithm is failed, when $S N R$ in $M$ processing blocks of signal is less than $S N R_{\min }$.

So, the $P_{d}$ for AWGN channels is changed to

$$
R F_{\text {fading }}=R F \times(1-\operatorname{Pr}(r \leq R))=R F \times\left(\exp \left(-\rho^{2}\right)\right) .
$$

From the above equation, it is obvious that the reliability factor of chip rate estimation algorithm reduces by the factor of $\exp \left(-\rho^{2}\right)$ when all $M$ processing windows have been faded.

Table 1 shows the $S N R_{\min }$ for achieveing a $R F$ greater than 0.99 in terms of spreading factor $(S F)$ over AWGN channels. These results are extracted using computer simulations. It can be seen, by $3 \mathrm{~dB}$ increase in $S F$, the $S N R_{\min }$ increases by $3 \mathrm{~dB}$.
Table 1. $S N R_{\min }$ for achieving a $R F$ greater than 0.99 in terms of $S F$ over AWGN channels.

\begin{tabular}{|l|l|l|l|l|l|l|}
\hline$S F[\mathrm{~dB}]$ & 15 & 18 & 21 & 24 & 27 & 30 \\
\hline$S N R_{\min }[\mathrm{dB}]$ & -5 & -8 & -11 & -14 & -17 & -20 \\
\hline
\end{tabular}

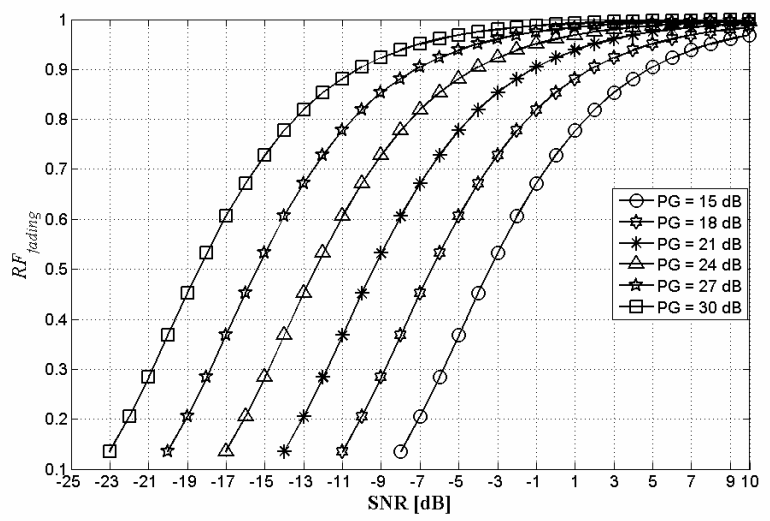

Figure 3. $R F_{\text {fading }}$ in terms of received SNR for different values of $S F$.

Figure 3 shows $R F$ in terms of SNR in flat fading channels with Rayleigh distribution for different values of $S F$. It can be seen that the $R F$ is greater than 0.95 for $\mathrm{SNR}=8 \mathrm{~dB}$ in $S F=15 \mathrm{~dB}$. It is obvious that the performance of the proposed algorithm degraded $13 \mathrm{~dB}$ with respect to that of AWGN channels.

Now, we propose a solution to overcome effect of fading channels on the performance of the proposed algorithm. If the distance between two neighboring antennas in eavesdropping receiver is large enough and communication environment is rich scattering, fading channels experienced by these antennas are independent. So, the reliability factor of the proposed chip rate estimation algorithm improves. So that

$$
R F_{\text {fading }_{m}}=R F \times\left(1-(\operatorname{Pr}(r \leq R))^{L}\right),
$$

where $R F_{\text {fading }_{m}}$ denotes reliability factor of the proposed chip rate estimation algorithm with $L$ receiving antennas in eavesdropping receivers over independent fading channels. Figure 4 shows $R F_{\text {fading }_{m}}$ in terms of SNR for spreading factor of $15 \mathrm{~dB}$ and different numbers for receiving antennas in eavesdropping receiver. It can be seen from this Figure that by increasing the number of receiving antennas from 1 to $4, R F_{\text {fading }_{m}}$ increases from 0.53 to 0.95 at $\mathrm{SNR}=-3 \mathrm{~dB}$.

In practice, assuming independent channel gains for receiving antennas is not realistic due to limitation size 
of the eavesdropping receiver. The correlation matrix of an antenna array depends not only on the array configuration but also on the incident angle of the incoming signal. Different parameters affect the correlation in different ways. Branch correlation depends on the antenna height and antenna separation through their ratio [11]. For a given antenna array, correlation between signals received by two neighbouring antennas varies with their corresponding angle of incidence so that it reaches its maximum value when the signal comes from the endfire direction and reduces gradually as the signal direction moves towards the broadside [12]. The covariance matrix among channel power gains e.g. $\left[\begin{array}{lll}\delta_{i, 1} & \cdots & \delta_{i, L}\end{array}\right]$ in the receiver is denoted by

$$
\Omega=\left[\begin{array}{cccc}
\omega_{1,1} & \omega_{1,2} & \cdots & \omega_{1, L} \\
\omega_{2,1} & \omega_{2,2} & \cdots & \omega_{2, L} \\
\vdots & \vdots & \ddots & \vdots \\
\omega_{L, 1} & \omega_{L, 2} & \cdots & \omega_{L, L}
\end{array}\right]_{L \times L} .
$$

$R F_{\text {corr-fading }}$, which is the reliability factor of the proposed chip rate estimation algorithm for correlated fading channel, is obtained using Bayes' theorem for correlated fading channels as follows

$$
R F_{\text {corr - fading }}=R F \times\left[1-\operatorname{Pr}(r \leq R)^{1+\sum_{j=2}^{L} \prod_{i=1}^{j-1}\left(1-\omega_{i, j}\right)}\right]
$$

In the above equation, $\omega_{i, j}$ s for $i \neq j$ are less than one. So $\left(1-\omega_{i, j}\right)$ and $\prod_{i=1}^{j-1}\left(1-\omega_{i, j}\right)$ is less than 1 . Therefore,

$$
\sum_{j=2}^{L} \prod_{i=1}^{j-1}\left(1-\omega_{i, j}\right) \leq L-1
$$

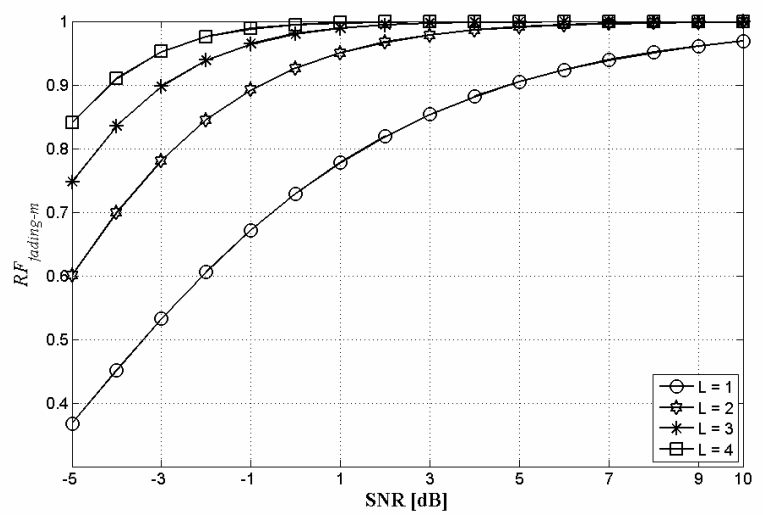

Figure 4. $R F_{\text {fading }_{m}}$ in terms of received SNR for different number of received antennas and $S F=15 \mathrm{~dB}$.

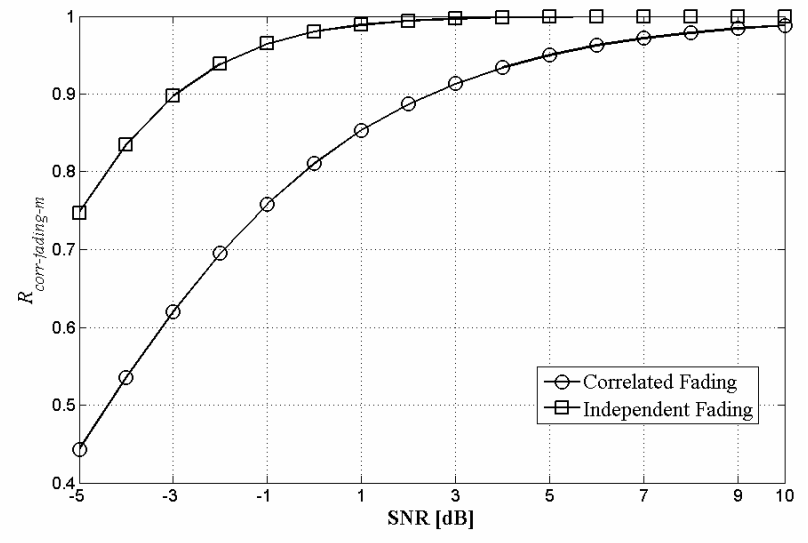

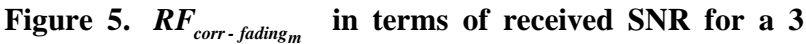
antenna array with correlated and independent fading channels and $\mathrm{SF}=15 \mathrm{~dB}$.

Therefore, the power of $\operatorname{Pr}(r \leq R)$ in equation (22) is less than $L$. In comparison to (20), it is obvious that, $R F_{\text {corr-fading }_{\mathrm{m}}}$ is less than $R F_{\text {fading }_{\mathrm{m}}}$.

Figure 5 shows $R F_{\text {corr-fading }_{\mathrm{m}}}$ in terms of received SNR for a 3 antenna array receiving correlated signals. Similar to that of [12], we assume triangular array at the eavesdropping receiver, located at the height of $30.43 \mathrm{~m}$, and its correlation matrix is given by

$$
\boldsymbol{\Omega}=\left[\begin{array}{ccc}
1 & 0.727 & 0.913 \\
0.727 & 1 & 0.913 \\
0.913 & 0.913 & 1
\end{array}\right]
$$

It can be seen from Figure 5, that $R F_{\text {corr-fading }}$ for triangular and antenna arrays is 0.62 at $\mathrm{SNR}=-3 \mathrm{~dB}$. It is obvious that $R F_{\text {corr-fading }_{\mathrm{m}}}$ decreases by 0.27 compared with $R F_{\text {fading }_{\mathrm{m}}}$ of an antenna array consisting of 3 antennas with independent fading channels.

\section{Simulation Results}

Computer simulations have been performed using two streams of spread spectrum signals with both BPSK spreading and data modulation. Gold sequences with lengths of 63 (user 1) and 31 (user 2) are considered as spreading sequences. Their chip rates are 20 Mega chips/sec and 40 Mega chips/sec, respectively. Processing window size is considered to be $160 \mu \mathrm{sec}$, and $M=$ 10 is the number of windows, which is used for averaging. Chip rates of each signal have been obtained using estimated impulse response of the channel. Bit rate of each signal has been estimated using the time difference 
between the successive equal amplitude peaks in the output of the correlation estimator.

Figure 6 shows fluctuations of the correlation estimator at SNR of $-5 \mathrm{~dB}$. The time differences between two successive equal amplitude peaks have been estimated $0.775 \mu \mathrm{sec}$ and $3.175 \mu \mathrm{sec}$.

Figure 7(a) shows the estimated channel impulse response for the spreading sequence with length of 63 and sampling frequency of $F_{s}=20 \mathrm{MHz}$. This signal has more SNR relative to the other user and produces the greatest eigenvalue in the estimated received signal covariance matrix; therefore, its first corresponding estimated eigenvector is selected for the chip rate estimation. A window of estimated channel impulse response as long as bit time $=3.175 \mu$ sec is considered for the chip rate estimation. Figure 7(b) shows the estimated, which is given by (9), and exact spreading sequence, it is obvious they are matched together. Figure 7(c) shows $d$ sgn $\left(h_{k, i}(t)\right) / d t$, it can be seen from this figure, zero crossing occurs when sign of signal changes.

Figure 8(a) shows that the distribution of zero crossing number for the $d \operatorname{sgn}\left(h_{k, i}(t)\right) / d t$ in terms of sampling frequency. Figure 8(b) shows the $Z C R$ of the $\left(h_{k, i}(t)\right) / d t$, which is defined in (11), in terms of sampling frequency. Figure 8(c) shows WZCR for $d$ sgn $\left(h_{k, i}(t)\right) / d t$, which is defined in (12), in terms of sampling frequency. It obvious maximum number of WZCR corresponds to the sampling frequency equal to Nyquist rate, which is equivalent to a chip rate $20 \mathrm{Mega}$ chips/sec. Hence, $T_{c}=1 /\left(20 \times 10^{6}\right)=50 \mathrm{nsec}$, code length is obtained as $T_{b} / T_{c}=3.15 \mu \mathrm{sec} / 50 \mathrm{nsec}=63$.

Figure 9(a) shows the probability of chip rate detection related to first user for 10000 times Monte Carlo test. It can be seen in this Figure, sampling frequency of 40 $\mathrm{MHz}$ is estimated by probability of more than $97.5 \%$ as a

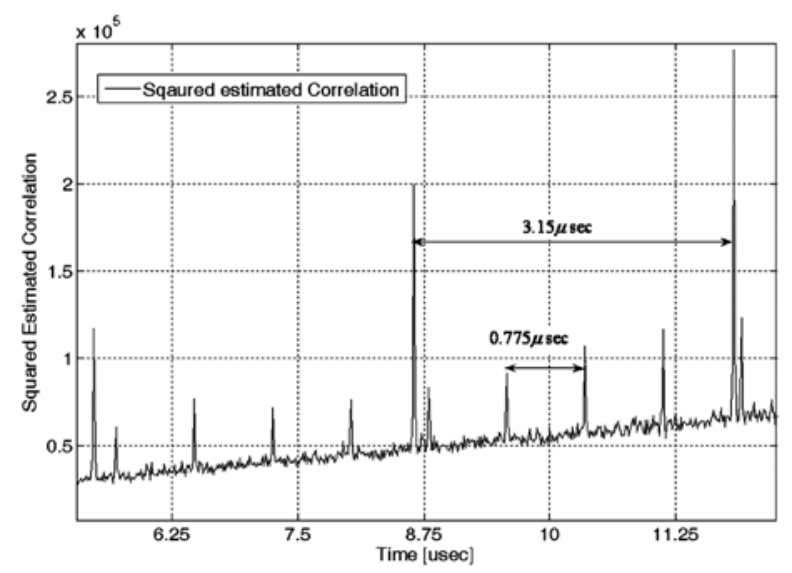

Figure 6. Fluctuations of correlation estimator output.
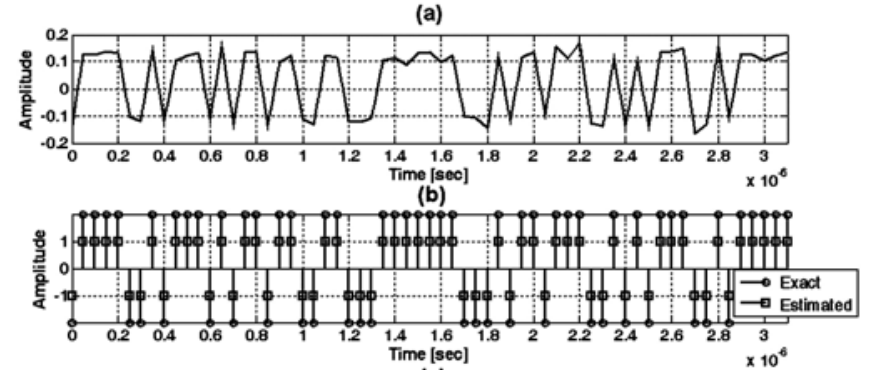

(c)

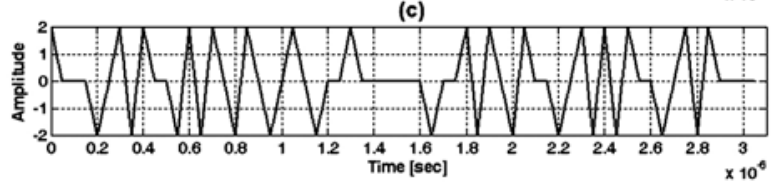

Figure 7. (a) Estimated eigenvector corresponding to the maximum eigenvalue of the received signal covariance matrix. (b) Exact and estimated spreading sequence of user 1. (c) The differential of the estimated eigenvector.
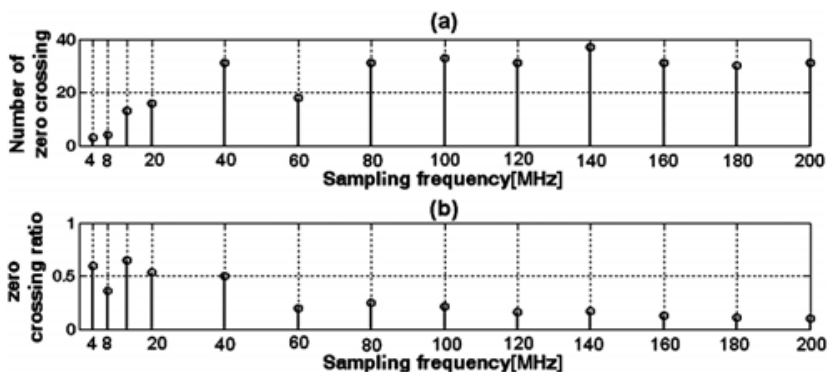

(c)

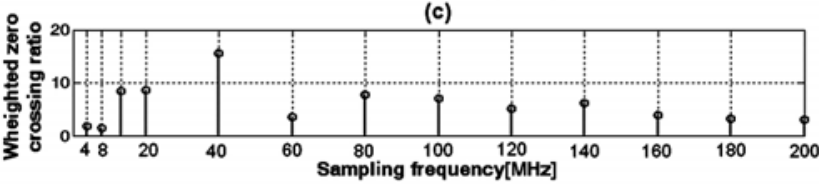

Figure 8. (a) Number of Zero crossings in terms of sampling frequency. (b) Zero crossing ratio in terms of sampling frequency. (c) weighted zero crossing ratio in terms of sampling frequency, all of them are related to user 1.

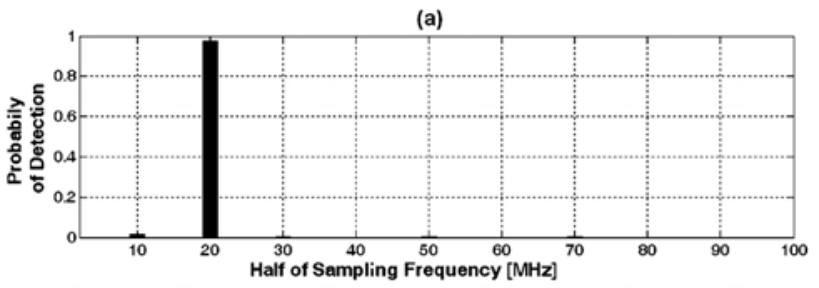

(b)

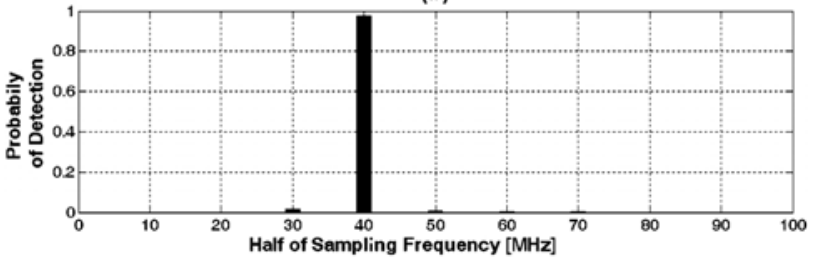

Figure 9. (a) Probability of chip rate detection related to the first user for 10000 times Monte Carlo Test. (b) Probability of chip rate detection related to the second user for 10000 times Monte Carlo Test. 
sampling frequency with maximum WZCR, which is equivalent to chip rate of 20 Mega chips/sec. Figure 9(b) is similar to Figure 9(a) except it is corresponds to second user. It can be seen sampling frequency of $80 \mathrm{MHz}$ is estimated by probability greater than $97 \%$ as a sampling frequency with maximum WZCR, which is equivalent to a chip rate 40 Mega chips/sec.

\section{Conclusions}

This paper considered the problem of blind chip rate estimation of direct sequence spread spectrum (DS-SS) signals in multi-rate and multi-user direct-sequence code division multiple accesses (DS-CDMA). The estimation is based on the multi-rate sampling of the estimated differential channel impulse response. Simulation results showed that the chip rate and bit rate can be determined exactly in very low SNR $(-5 \mathrm{~dB})$ and in multi-rate and multi-user field. Therefore, it is possible to blindly estimate the spreading factor of each user. In fading channels, the reliability factor of the proposed chip rate estimation algorithm is analyzed and a receiver with multiple antennas is proposed to improve the reliability factor of the proposed algorithm.

\section{References}

[1] E. Dahlman, P. Beming, J. Knutsson, F. Ovesjo, M. Persson, and C. Roobol, "WCDMA-the radio interface for future mobile multimedia communications," IEEE Transactions on Vehicular Technology, Vol. 47, pp. 1105-1118, November 1998.

[2] P. Taaghol, B. G. Evans, E. Buracchini, G. De Gaudinaro, Joon Ho Lee, and Chung Gu Kang, "Satellite UMTS/ IMT2000 W-CDMA air interfaces,” IEEE Communications Magazine, Vol. 37, pp. 116-126, September 1999.

[3] Z. Zhang, L. Li, and X. Xiao; "Carrier frequency and chip rate estimation based on cyclic spectral density of MPSK signals," in Proceedings of Communications, Circuits and Systems Conference, Vol. 2, pp. 859 -862, June 2004.

[4] W. J. Ma, S. M. Yang, W. Ren, Z. H. Xue, and W. M. Li, "Spectral correlation function in low SNR environment," in Proceedings of Radio Science Asia-Pacific Conference, pp. 197-200, August 2004.

[5] Y. X. Li, M. Yi, Q. Yang, X. C. Xiao, and H. M. Tai, "Low SNR BPSK signal chip rate estimation using a wavelet based spectral correlation algorithm," in Proceedings of Circuit and Systems Conference, Vol. 3, pp. 247-249, August 2002.

[6] P. G. Turner, R. Hewitt, and D. J. Purle, "Chip-rate processing for software defined radios,” in Proceedings of 3G Mobile Communication Technologies, pp. 292-296, March 2001.

[7] Y. Jin and H. B. Ji, “A cyclic-cumulant based method for DS-SS signal detection and parameter estimation,” in Proceedings of Microwave, Antenna, Propagation and EMC Technologies for Wireless Communications Conference, Vol. 2, pp. 966-969, August 2005.

[8] S. Ghavami and B. Abolhassani, "Blind chip time estimation in multirate CDMA transmissions using multirate sampling in slow flat fading channels," in the Proceedings of IEEE-ICCS08, pp. 1344-1348, November 2008.

[9] C. N. Nzéza, R. Gautier, and G. Burel, "Parallel blind multiuser synchronization and sequence estimation in multirate CDMA transmissions," in Proceedings of IEEE - ACSSC, pp. 2157-2161, November 2006.

[10] C. N. Nzéza, R. Gautier, and G. Burel, "Blind multiuser detection in multirate CDMA transmissions using fluctuations of correlation estimators," in Proceedings of IEEE - Globecom, pp. 1-5, November 2006.

[11] Q. T. Zhang, "Maximal-ratio combining over Nakagami fading channels with an arbitrary branch covariance matrix,” IEEE Transactions on Vehicular Technology, Vol. 48, No. 4, pp.1141-1150, July 1999.

[12] W. C. Lee, "Mobile communications: Design fundamentals,” 2nd Edition, New York, Wiley, pp. 202-211, 1993. 